Article

\title{
Creating Diversified Response Profiles from a Single Quenchometric Sensor Element by Using Phase-Resolved Luminescence
}

\author{
Elizabeth C. Tehan ${ }^{1}$, Rachel M. Bukowski ${ }^{1}$, Vamsy P. Chodavarapu ${ }^{2, *}$, Albert H. Titus ${ }^{3}$, \\ Alexander N. Cartwright ${ }^{3}$ and Frank V. Bright ${ }^{1, *}$
}

1 Department of Chemistry, Natural Sciences Complex, University at Buffalo, The State University of New York, Buffalo, NY 14260, USA; E-Mails: cornell.elizabeth@yahoo.com (E.C.T.); rmbukowski@gmail.com (R.M.B.)

2 Department of Electrical and Computer Engineering, McGill University, McConnell Engineering Building, 3480 University Street, Montreal, QC H3A 0E9, Canada

3 Department of Electrical Engineering, Bonner Hall, University at Buffalo, The State University of New York, Buffalo, NY 14260, USA; E-Mails: ahtitus@buffalo.edu (A.H.T.); anc@buffalo.edu (A.N.C.)

* Authors to whom correspondence should be addressed;

E-Mails: vamsy.chodavarapu@mcgill.ca (V.P.C.); chefvb@buffalo.edu (F.V.B.);

Tel.: +514-398-3118 (V.P.C.); +716-645-6800 (ext. 2162) (F.V.B.);

Fax: +514-398-4470 (V.P.C.); +716-645-6963 (F.V.B.).

Academic Editor: W. Rudolf Seitz

Received: 16 November 2014 / Accepted: 12 December 2014 / Published: 5 January 2015

\begin{abstract}
We report a new strategy for generating a continuum of response profiles from a single luminescence-based sensor element by using phase-resolved detection. This strategy yields reliable responses that depend in a predictable manner on changes in the luminescent reporter lifetime in the presence of the target analyte, the excitation modulation frequency, and the detector (lock-in amplifier) phase angle. In the traditional steady-state mode, the sensor that we evaluate exhibits a linear, positive going response to changes in the target analyte concentration. Under phase-resolved conditions the analyte-dependent response profiles: (i) can become highly non-linear; (ii) yield negative going responses; (iii) can be biphasic; and (iv) can exhibit super sensitivity (e.g., sensitivities up to 300 fold greater in comparison to steady-state conditions).
\end{abstract}


Keywords: phase-resolved luminescence detection; quenchometric sensor; gaseous oxygen $\left(\mathrm{O}_{2}\right)$ sensing

\section{Introduction}

There has been significant effort devoted to creating optical chemical sensors for a wide variety of target analytes [1-5]. There have also been simultaneous and largely related efforts to create ensembles of sensors that act independently and provide unique responses for the same target analyte (i.e., diversified responses; 1 analyte, $n$ sensor elements, $n>>1)$ ) and this concept has also been extended to simultaneous multi-analyte detection ( $m$ analytes, $n$ sensor elements; $n>m$ ) [3,6-11]. In all previous efforts, researchers have created sensor ensembles and thus diversified responses by adjusting the: (a) individual recognition elements within the analyte-permeable host matrix and/or (b) host matrix to control the partitioning of the target analyte into the host matrix and eventual interaction with the recognition element, as illustrated in Figure 1 [12-14].

\section{Tune Recognition Element}

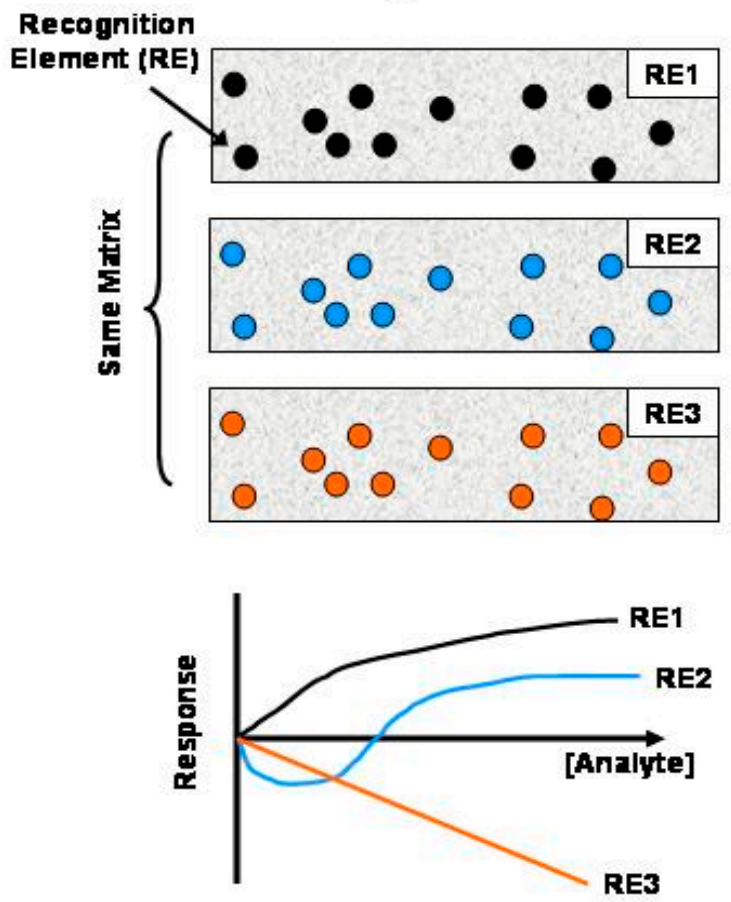

\section{Tune Host Matrix}
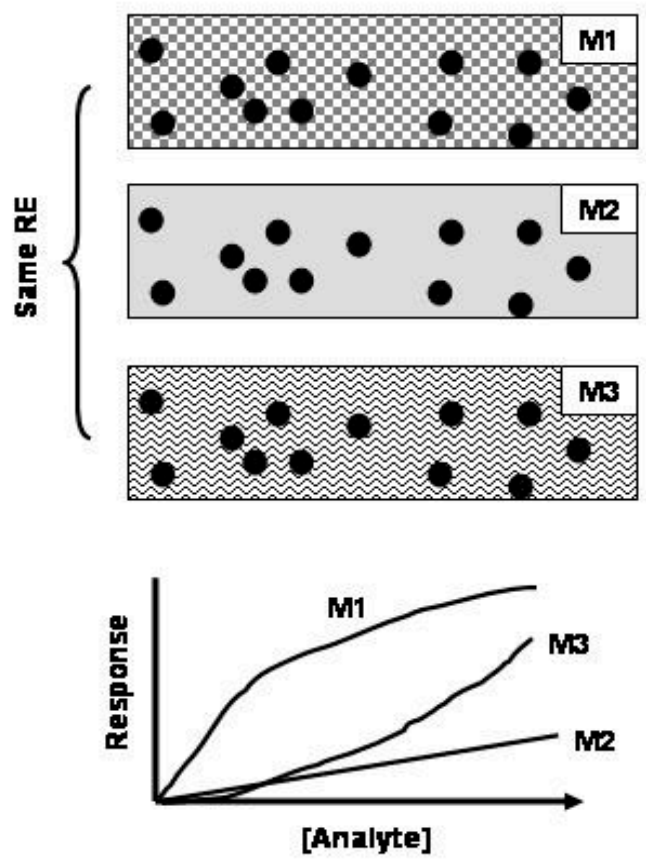

Figure 1. Illustration that describes traditional approaches of creating chemical sensors with a range of response profiles for the same target analyte. Three hypothetical sensor elements are shown under each approach. Each sensor yields a unique response profile for the intended target analyte that depends on the recognition element $\left(\mathrm{RE}_{x}\right)$ or matrix $\left(\mathrm{M}_{x}\right)$.

In this paper, we describe a strategy for creating a continuously tunable response from a single sensor element. The approach exploits a unique feature of phase-resolved luminescence [15]. The potential of this strategy is demonstrated for a single $\mathrm{O}_{2}$-responsive xerogel-based sensor element as it offers a simple and well understood behavior. Consider the following scenario: (i) a single type of 
luminophore molecule sequestered within a quencher-permeable host matrix; (ii) these luminophore molecules emit from largely similar microenvironments; and (iii) the quencher molecules have similar accessibilities to the individual luminophore molecules. Under steady-state illumination conditions, one can write the Stern-Volmer relationship [16] given by Equation (1):

$$
I_{0} / I=1+K \mathrm{SV}[\mathrm{Q}]
$$

where in this expression, [Q] is the concentration of quencher, $I_{0}$ and $I$ represent the steady-state luminescence intensities in the absence and presence of quencher, Q, respectively, and $K_{\mathrm{SV}}$ is the Stern-Volmer quenching constant. Under dynamic quenching conditions, $K_{\mathrm{SV}}$ depends on $k_{\mathrm{Q}}$, the bimolecular quenching constant that describes the luminophore and quencher interaction and $\tau$, the luminophore excited-state luminescence lifetime in the absence of any quencher molecules (i.e., $\left.K \mathrm{sv}=k_{\mathrm{Q}} \tau_{0}\right)[16]$.

If we electronically excite (ex) the luminophores within this same sensor element by using sinusoidally modulated electromagnetic radiation (Figure 2A) at frequency $(f)$, the resulting emission (em) will be phase shifted $(\theta)$ and demodulated $(M)$ in comparison to the excitation by an extent that depends on the luminophore excited-state lifetime $(\tau)[17,18]$ :

$$
\begin{gathered}
\theta=\arctan (2 \pi f \tau) \\
M=\left[1+(2 \pi f)^{2} \tau^{2}\right]^{-1 / 2}
\end{gathered}
$$

In turn, we can write expressions for $I$ and $\tau$ at any $\mathrm{Q}$ concentration as:

$$
\begin{gathered}
I=I_{0} /\left[1+\left(k_{\mathrm{Q}} \tau_{0}[\mathrm{Q}]\right)\right] \\
\tau=1 /\left[1 / \tau_{0}+k_{\mathrm{Q}}[\mathrm{Q}]\right]
\end{gathered}
$$

If we record the luminescence from this same sensor element using a phase-sensitive detector (i.e., a lock-in amplifier) (Figure 2B,C) [15], we can write an expression for the phase-sensitive luminescence intensity (PSLI) as Equation (6):

$$
\operatorname{PSLI}\left(\theta_{\mathrm{D}}\right)=I M \cos \left(\theta_{\mathrm{D}}-\theta\right)
$$

where $I$ denotes the steady-state luminescence intensity, $M$ represents the demodulation factor, $\theta_{D}$ is the detector (i.e., lock-in amplifier) phase angle, and $\theta$ is the luminescence phase angle (all terms depend on Q). If we substitute Equations (2)-(5) into Equation (6), we can write an expression for the phase-sensitive analog of Equation (1), namely as:

$$
\text { PSLI } 0 / \text { PSLI }=\left[I_{0} M_{0} \cos \left(\theta_{\mathrm{D}}-\theta_{0}\right)\right] /\left[I M \cos \left(\theta_{\mathrm{D}}-\theta\right)\right]
$$

Equation (7) provides the key relationship between the fundamental properties of the sensor element (i.e., $k_{\mathrm{Q}}, \tau_{0}$, and $I_{0}$ ) and the phase-sensitive analog of $I_{0} / I$ (i.e., PSLI $0 / \mathrm{PSLS}$ ) as a function of [Q], $\theta_{\mathrm{D}}$, and $f$. Inspection of Equation (7), suggests a strategy for tuning the response profiles (i.e., creating diversity) from a single sensor element by adjusting $\theta_{\mathrm{D}}$ and $f$. 


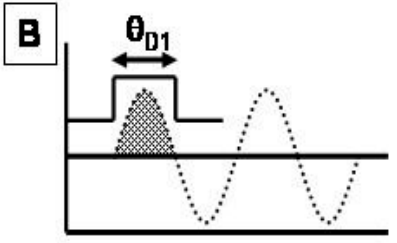

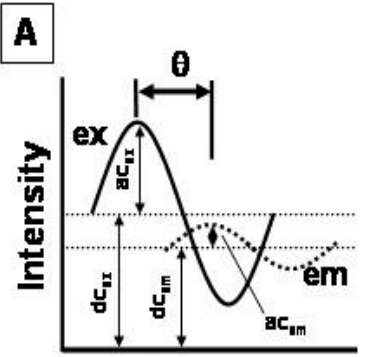

Time
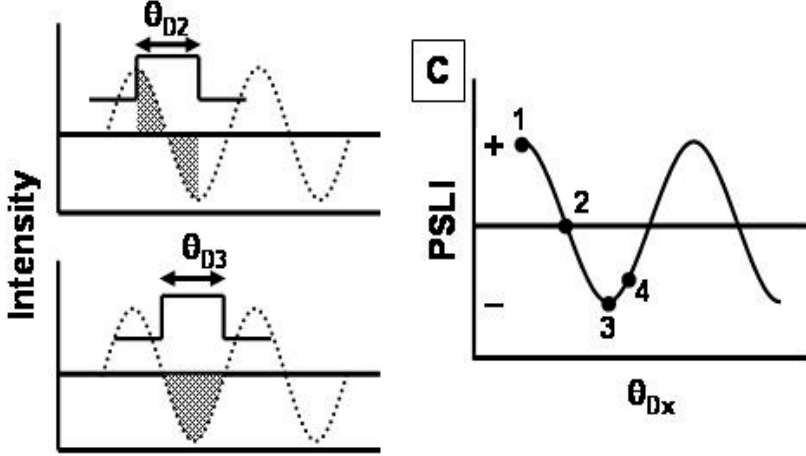

$M=\left[a_{e m} / d c_{e m}\right]\left[a_{e x} d d c_{e x}\right]$

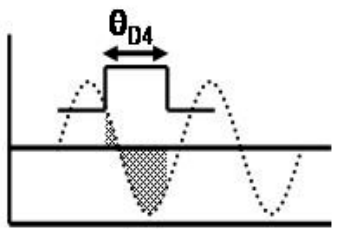

Time

Figure 2. A basic phase resolved experiment. (A) Phase-modulation concept. Excitation (ex), emission (em), luminescence phase shift $(\theta)$, and luminescence demodulation factor $(M)$ are shown; (B) The phase resolution experiment with the detector phase angle $\left(\theta_{\mathrm{Dx}}\right)$ set at four different values $\left(\theta_{\mathrm{D} 1}\right.$ to $\left.\theta_{\mathrm{D} 4}\right)$. The shaded region denotes the area under the modulated emission that is integrated by the $\pi$ function; (C) The phase-sensitive luminescence intensity (PSLI) that results from the different $\theta_{\text {Dx }}$ settings shown in (B).

\section{Experimental Section}

\subsection{Chemical Reagents}

Tris(4,7'-diphenyl-1,10'-phenanathroline) ruthenium(II) chloride pentahydrate $\left(\left[\operatorname{Ru}(\mathrm{dpp})_{3}\right]^{2+}\right)$ was purchased from GFS Chemicals, Inc. (Powell, OH, USA) and purified as described in the literature [19]. Tetraethyorthosilane (TEOS) and n-octyltriethoxysilane (C8-TEOS) were purchased from Gelest, Inc. (Morrisville, PA, USA). HCl was obtained from Fisher Scientific Co. (Pittsburgh, PA, USA). EtOH was a product of Quantum Chemical Corp. (New York, NY, USA). Deionized water was prepared to a specific resistivity of at least $18 \mathrm{M} \Omega$-cm by using a Barnstead NANOpure ${ }^{\circledR}$ II system.

\subsection{Preparation of $\left[R u(d p p)_{3}\right]^{2+}$-Doped Xerogel Sensing Films}

The sol solutions were prepared as described elsewhere [20]. Xerogel films were formed directly onto the surface of a light emitting diode (LED, $\lambda_{\max }=468 \mathrm{~nm}$, Stanley Electric Co., Inc., (Tokyo, Japan). The LED surface was cleaned first by rinsing with $1 \mathrm{M} \mathrm{NaOH}$ then with copious amounts of deionized water and $\mathrm{EtOH}$, and allowed to dry under ambient conditions. Films were formed by depositing $20 \mu \mathrm{L}$ of sol solution on to the LED surface. Xerogel-coated LEDs were stored in the dark under ambient conditions for at least seven days before evaluation. Previous research [20] has shown that 
these class II xerogel-based sensor elements exhibit linear $I_{0} / I v s . \mathrm{O}_{2}$ plots and the responses are stable for several years.

\subsection{Instrumentation}

Figure 3 presents a simplified schematic of the phase-resolved luminescence system that was constructed for this research. A model DS345 function generator (Stanford Research Systems, Sunnyvale, CA, USA) provided the AC and DC components to drive the LED. The function generator output also served as the reference input to a Stanford Research Systems (model SR830) lock-in amplifier. The xerogel-coated LED was enclosed within an optically transparent housing with an inlet/outlet for gas flow. In the current experiments, the quencher was gaseous $\mathrm{O}_{2}$. The emission was monitored through a $570 \mathrm{~nm}$ long pass optical filter (Oriel, Irvine, CA, USA) and detected by a $1 \mathrm{MHz}$ bandwidth silicon photodiode (Thorlabs, Inc., Newton, NJ, USA). The photodiode output signal was directed to the lock-in amplifier input. The lock-in amplifier served to record the phase sensitive luminescence intensity (PSLI) at a given detector phase angle setting $\left(\theta_{\mathrm{D}}\right)$.

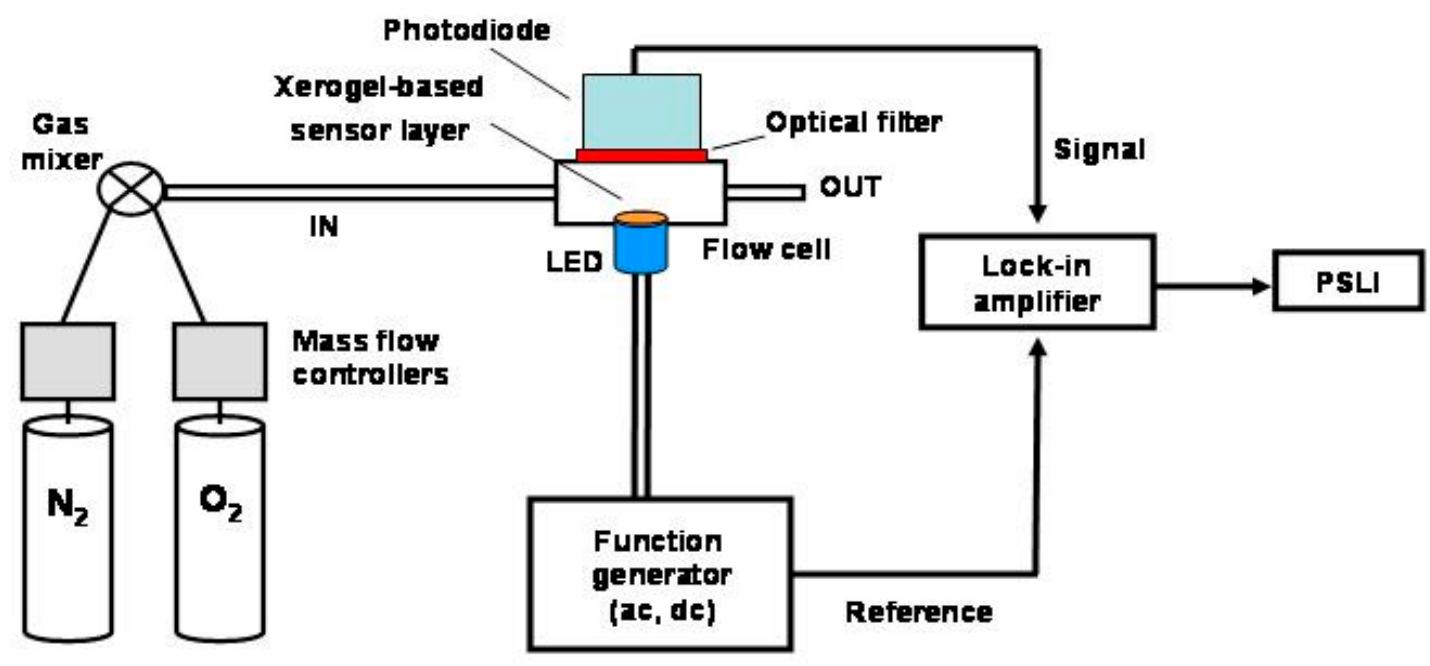

Figure 3. Simplified schematic of the phase-sensitive instrument used in this research. The modulation frequency $(f)$ is controlled by the function generator, the detector phase angle $\left(\theta_{\mathrm{D}}\right)$ is adjusted with the lock-in amplifier, and the sample composition that reaches the sensor element is controlled by the mass flow controllers.

In a typical experiment, we adjust the excitation modulation frequency $(f)$ to a given value (20 or $50 \mathrm{kHz}$ ) and increment $\theta_{\mathrm{D}}$ in $22.5^{\circ}$ steps from $0^{\circ}$ to $180^{\circ}$. We then systematically adjust the environment surrounding the sensor from pure $\mathrm{N}_{2}$ to pure $\mathrm{O}_{2}$ by using a pair of precision mass flow controllers (model PC1NP1U1V_1A, Sensirion Inc. (Westlake Village, CA, USA). Equilibrium is evident when the luminescence intensity remains constant to within $\pm 2 \%$. There was no detectable sensor response hysteresis or photo-bleaching. The $\mathrm{O}_{2}$ concentrations were accurate to $\pm 0.8 \%$ between $10 \%$ and $100 \%$ full scale and to $\pm 0.08 \%$ below $10 \%$ full scale.

The $\theta_{D^{-}}, f-$, and $\mathrm{O}_{2}$-dependent phase-resolved Stern-Volmer plots were then constructed. Sensors were prepared in triplicate and replicates were prepared using at least three reagent batches on different 
days. All experiments were performed in at least triplicate over the course of several months. Results are reported as the mean for all measurements under a given set of conditions \pm one standard deviation.

\section{Results and Discussion}

Figures 4 and 5 present simulated (A and B) and experimental ( $C$ and $D)$ results for the sensor film when modulated at 20 and $50 \mathrm{kHz}$, respectively, as a function of $\mathrm{O}_{2}$ (quencher) concentration and $\theta_{\mathrm{D}}$. In the simulations we assumed: $\tau_{0}=5.0 \mu \mathrm{s}$ and $K_{\mathrm{SV}}=0.1 \mathrm{O}_{2} \%^{-1}$. Figures $4 \mathrm{~A}$ and $5 \mathrm{~A}$ show the simulated responses at each $\theta_{\mathrm{D}}$. Figures $4 \mathrm{~B}$ and $5 \mathrm{~B}$ show the simulated response with the highest responses $\left(\theta_{\mathrm{D}}=90^{\circ}\right.$ in Figure $4 \mathrm{~A}, \theta_{\mathrm{D}}=67.5^{\circ}$ in Figure $\left.5 \mathrm{~A}\right)$ removed. Figures $4 \mathrm{C}$ and $5 \mathrm{C}$ show the experimental responses at each $\theta_{\mathrm{D}}$. Figures $4 \mathrm{D}$ and $5 \mathrm{D}$ show the experimental responses with the highest responses $\left(\theta_{\mathrm{D}}=90^{\circ}\right.$ in Figure $4 \mathrm{C}, \theta_{\mathrm{D}}=67.5^{\circ}$ in Figure $\left.5 \mathrm{C}\right)$ removed.
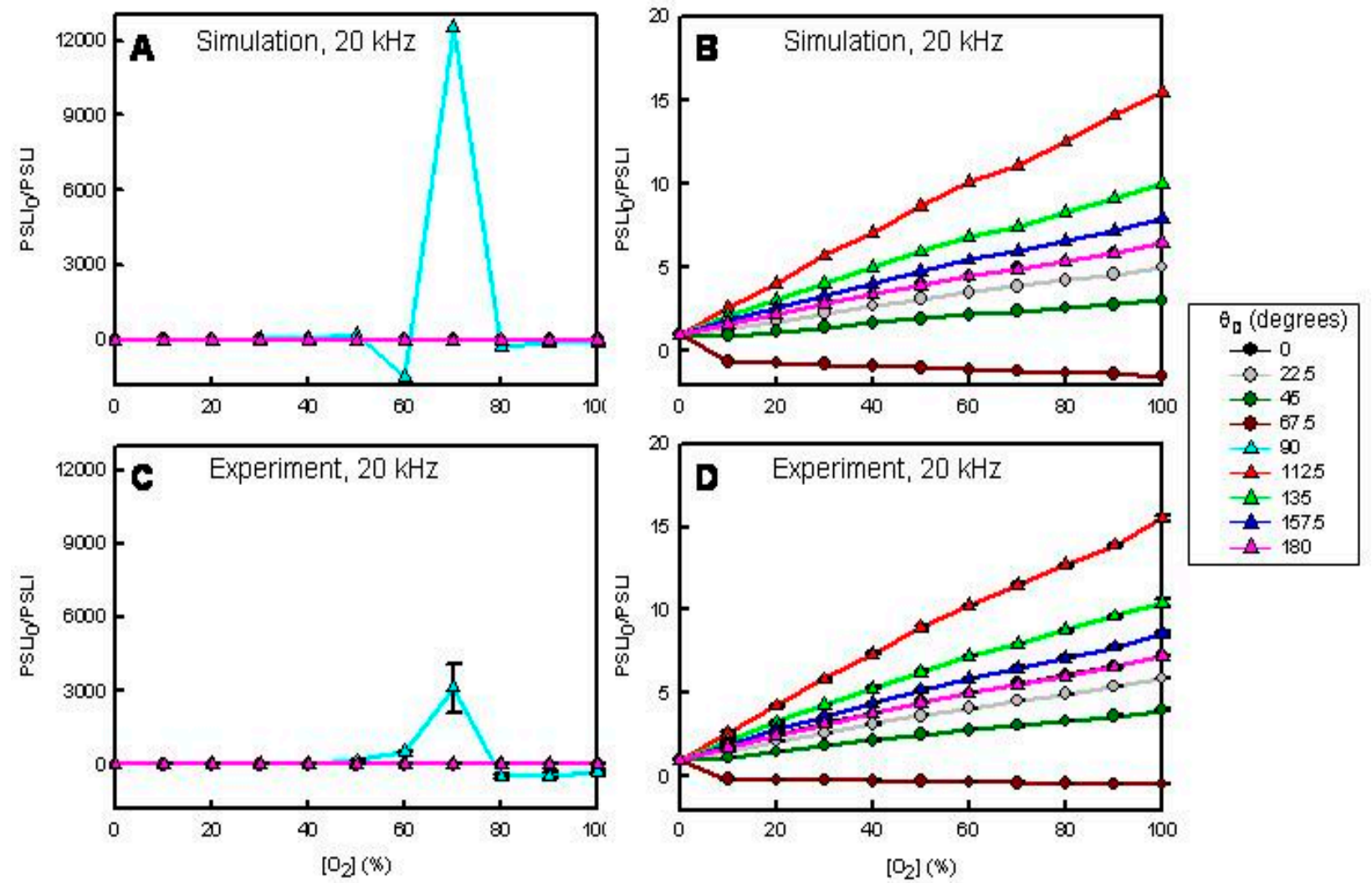

Figure 4. Simulated (A,B) and experimental (C,D) $\mathrm{O}_{2}$-dependent, phase sensitive Stern-Volmer plots for a $\left[\mathrm{Ru}(\mathrm{dpp})_{3}\right]^{2+}$-doped C8-TEOS/TEOS-based xerogel film at $f=20 \mathrm{kHz}$. In the simulations $\tau_{0}=5.0 \mu \mathrm{s}$ and $K_{\mathrm{SV}}=0.1 \mathrm{O}_{2} \%^{-1}$. (B,,D) $\theta_{D}=90^{\circ}$ data omitted.

Inspection of these data sets shows several interesting results. First, the simulations and experiments agree very well. Second, the response profiles depend on $f$ and $\theta_{D}$. Thus, one can tune the sensor response and sensitivity simply by adjusting $f$ or $\theta_{D}$. In all previous sensor research, there was no provision for achieving a diverse response from a single sensor element (cf., Figure 1). Third, the response profiles are sometimes non-linear. In a steady-state experiment this sensor element exhibits a linear response with a $K_{\mathrm{SV}}$ of $\sim 0.1 \mathrm{O}_{2} \%^{-1}$ [20]. Fourth, there are conditions when the phase-resolved 
Stern-Volmer plots exhibit negative going responses due to negative phase shift change. Fifth, there are conditions when the phase-resolved Stern-Volmer plots exhibit biphasic response profiles. Finally, there are conditions when the response (PSLIo/PSLI) is ultra-sensitive. For example, in our previous work, the $I_{0} / I$ for $70 \% \mathrm{O}_{2}$ is $\sim 8$ for this sensor element [20]. In Figure $4 \mathrm{C}$ we show a reproducible response in excess of 3000 at $20 \mathrm{kHz}$ with a $\theta_{D}$ of $90^{\circ}$. Similarly, at $50 \mathrm{kHz}$ with a $\theta_{D}$ of $67.5^{\circ}$ we see a $\mathrm{PSLI}_{0} / \mathrm{PSLI}$ at $100 \% \mathrm{O}_{2}$ of $\sim 50$ whereas the $\mathrm{I}_{0} / \mathrm{I}$ at $100 \% \mathrm{O}_{2}$ is $\sim 10$ for this same sensor [20].
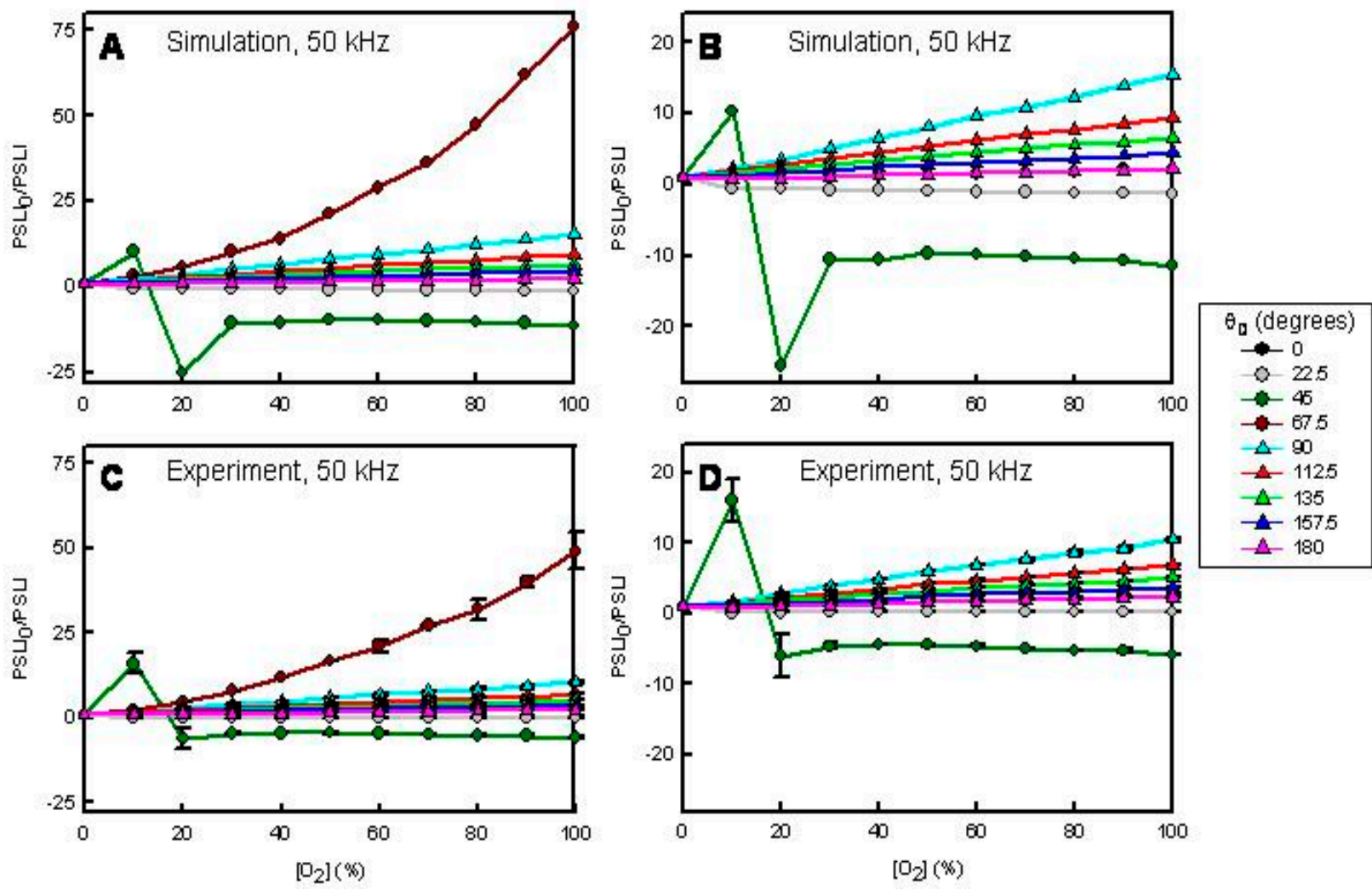

Figure 5. Simulated (A,B) and experimental (C,D) $\mathrm{O}_{2}$-dependent, phase sensitive Stern-Volmer plots for a $\left[\mathrm{Ru}(\mathrm{dpp})_{3}\right]^{2+}$-doped C8-TEOS/TEOS-based xerogel film at $f=50 \mathrm{kHz}$. In the simulations $\tau_{0}=5.0 \mu \mathrm{s}$ and $K_{\mathrm{SV}}=0.1 \mathrm{O}_{2} \%{ }^{-1}$. (B,D) $\theta_{D}=67.5^{\circ}$ data omitted.

\section{Conclusions}

We report on a simple and new approach for creating diversified sensor responses from a single sensor element. The approach is not only limited to quenchometric-based sensing, but also will work under any situation where there is a change in a luminophore excited state lifetime caused by the target analyte [21-25]. Phase-sensitive detection also leads to unique analyte-dependent response profiles that are not realized under previously used detection formats. These diversified responses are ideal for training artificial neural networks (ANNs) to identify patterns and features for improving the accuracy and precision of analyte concentration determinations [26]. This strategy of creating a diversified response from a single sensor element could also enable novel miniaturized sensor systems with built-in self-testing when integrated with a tunable multi-frequency phase fluorometric spectroscopic systems [27]. 


\section{Acknowledgments}

This work was supported in part by the United States National Science Foundation (NSF) and Canada Natural Sciences and Engineering Research Council (NSERC).

\section{Author Contributions}

F.V.B. conceived and designed the experiments; E.C.T., R.M.B. and V.P.C. performed the experiments; E.C.T. and R.M.B. analyzed the data; A.H.T. and A.N.C. contributed experimental and analysis tools; E.C.T., R.M.B., V.P.C., A.H.T., A.N.C. and F.V.B. wrote the paper.

\section{Conflicts of Interest}

The authors declare no conflict of interest.

\section{References}

1. Narayanaswamy, R.; Wolfbeis, O.S. Optical Sensors: Industrial, Environmental and Diagnostic Applications; Springer: Berlin, Germany, 2004.

2. Wang, X.D.; Wolfbeis, O.S. Optical methods for sensing and imaging oxygen: Materials, spectroscopies and applications. Chem. Soc. Rev. 2014, 43, 3666-3761.

3. Askim, J.R.; Mahmoudi, M.; Suslick, K.S. Optical sensor arrays for chemical sensing: The optoelectronic nose. Chem. Soc. Rev. 2013, 42, 8649-8682.

4. Wang, X.D.; Wolfbeis, O.S. Fiber-Optic Chemical Sensors and Biosensors (2008-2012). Anal. Chem. 2013, 85, 487-508.

5. Wolfbeis, O.S.; Weidgans, B.M. Fiber optic chemical sensors and biosensors: A view back. Nato Sci. Ser. II Math. 2006, 224, 17-44.

6. Wilson, M.S.; Nie, W.Y. Electrochemical multianalyte immunoassays using an array-based sensor. Anal. Chem. 2006, 78, 2507-2513.

7. Heo, J.; Crooks, R.M. Microfluidic biosensor based on an array of hydrogel-entrapped enzymes. Anal. Chem. 2005, 77, 6843-6851.

8. Albert, K.J.; Lewis, N.S.; Schauer, C.L.; Sotzing, G.A.; Stitzel, S.E.; Vaid, T.P.; Walt, D.R. Cross-reactive chemical sensor arrays. Chem. Rev. 2000, 100, 2595-2626.

9. Diehl, K.L.; Anslyn, E.V. Array sensing using optical methods for detection of chemical and biological hazards. Chem. Soc. Rev. 2013, 42, 8596-8611.

10. Rakow, N.A.; Suslick, K.S. A colorimetric sensor array for odour visualization. Nature 2000, 406, 710-713.

11. Nagl, S.; Wolfbeis, O.S. Optical multiple chemical sensing: Status and current challenges. Analyst 2007, 132, 507-511.

12. Chodavarapu, V.P.; Bukowski, R.M.; Kim, S.J.; Titus, A.H.; Cartwright, A.N.; Bright, F.V. Multi-sensor system based on phase detection, an LED array, and luminophore-doped xerogels. Electron. Lett. 2005, 41, 1031-1033. 
13. Daivasagaya, D.S.; Yao, L.; Yung, K.Y.; Hajj-Hassan, M.; Cheung, M.C.; Chodavarapu, V.P.; Bright, F.V. Contact CMOS imaging of gaseous oxygen sensor array. Sens. Actuators B Chem. 2011, 157, 408-416.

14. Chodavarapu, V.P.; Bukowski, R.M.; Titus, A.H.; Cartwright, A.N.; Bright, F.V. CMOS integrated luminescence oxygen multi-sensor system. Electron. Lett. 2007, 43, 688-689.

15. Mcgown, L.B.; Bright, F.V. Phase-Resolved Fluorescence in Chemical-Analysis. Crit. Rev. Anal. Chem. 1987, 18, 245-298.

16. Lakowicz, J.R. Principle of Fluorescence Spectroscopy: Second Edition; Kluwer Academic/Plenum Publishers: New York, NY, USA, 1999.

17. Gratton, E.; Jameson, D.M.; Hall, R.D. Multifrequency Phase and Modulation Fluorometry. Annu. Rev. Biophys. Bioeng. 1984, 13, 105-124.

18. Jameson, D.M.; Gratton, E.; Hall, R.D. The Measurement and Analysis of Heterogeneous Emissions by Multifrequency Phase and Modulation Fluorometry. Appl. Spectrosc. Rev. 1984, 20, $55-106$.

19. Lin, C.T.; Bottcher, W.; Chou, M.; Creutz, C.; Sutin, N. Mechanism of Quenching of Emission of Substituted Polypyridineruthenium(Ii) Complexes by Iron(Iii), Chromium(Iii), and Europium(Iii) Ions. J. Am. Chem. Soc. 1976, 98, 6536-6544.

20. Tao, Z.Y.; Tehan, E.C.; Tang, Y.; Bright, F.V. Stable sensors with tunable sensitivities based on class II xerogels. Anal. Chem. 2006, 78, 1939-1945.

21. Lakowicz, J.R. Principle of Fluorescence Spectroscopy: Third Edition; Springer: New York, NY, USA, 2006.

22. McGaughey, O.; Ros-Lis, J.V.; Guckian, A.; McEvoy, A.K.; McDonagh, C.; MacCraith, B.D. Development of a fluorescence lifetime-based sol-gel humidity sensor. Anal. Chim. Acta 2006, 570, 15-20.

23. Pickup, J.C.; Hussain, F.; Evans, N.D.; Rolinski, O.J.; Birch, D.J.S. Fluorescence-based glucose sensors. Biosens. Bioelectron. 2005, 20, 2555-2565.

24. Collier, B.B.; McShane, M.J. Time-resolved measurements of luminescence. J. Lumin. 2013, 144, 180-190.

25. Bukowski, R.M.; Chodavarapu, V.P.; Titus, A.H.; Cartwright, A.N.; Bright, F.V. Phase fluorometric glucose biosensor using oxygen as transducer and enzyme-doped xerogels. Electron. Lett. 2007, 43, 202-204.

26. Tang, Y.; Tao, Z.Y.; Bukowski, R.M.; Tehan, E.C.; Karri, S.; Titus, A.H.; Bright, F.V. Tailored xerogel-based sensor arrays and artificial neural networks yield improved $\mathrm{O}_{2}$ detection accuracy and precision. Analyst 2006, 131, 1129-1136.

27. Chodavarapu, V.P.; Daivasagaya, D.S. Methods and Devices for Xerogel based Sensors. U.S. Patent 2014/0080225 A1, 20 March 2014.

(C) 2015 by the authors; licensee MDPI, Basel, Switzerland. This article is an open access article distributed under the terms and conditions of the Creative Commons Attribution license (http://creativecommons.org/licenses/by/4.0/). 